\title{
Concussion knowledge and attitudes among amateur South African rugby players
}

C T Viljoen, ${ }^{\mathbf{1}}$ B Phys T, MSc Physiotherapy, M Schoeman, ${ }^{2}$ BSc (Human Movement Sciences), BSc (Hons) (Biokinetics), PhD (Biomechanics), C Brandt, ${ }^{1}$ BSc Physiotherapy, MSc Physiotherapy, PhD Physiotherapy, J Patricios, ${ }^{3}$ MBBCh, MMedSci, FACSM, FFSEM (UK), FFIMS, C van Rooyen, ${ }^{2}$ BCom, MCom

${ }^{1}$ Department Physiotherapy, University of the Free State, South Africa ${ }^{2}$ Department Biostatistics, University of the Free State, South Africa 3 Section Sports Medicine, Faculty of Health Sciences, University of Pretoria, and Department of Emergency Medicine, Faculty of Health Sciences, University of the Witwatersrand, South Africa

\section{Corresponding author: C T Viljoen (viljoen.ct@gmail.com)}

Background: The South African Rugby Union's BokSmart programme currently educates coaches and referees on concussion. Rugby players are often more familiar with their teammates than the coach or referee. Therefore they are wellpositioned to play a pivotal role in rugby safety if they have adequate knowledge to identify subtle signs and abnormal behaviour displayed by a concussed teammate. However, no programme focuses on concussion education among South African rugby players and there is a dearth of literature on concussion education programmes among rugby players which could lead to safer return to play (RTP) habits.

Objectives: To evaluate South African rugby players' concussion knowledge and attitudes/behaviours regarding RTP following a concussion.

Methods: A descriptive, cross-sectional study was used. Participants $(n=294)$ were divided into junior amateur high school (JAHS) $(n=216)$ and senior amateur club (SAC) $(n=78)$ players. The modified RoCKAS-ST questionnaire was used to evaluate their concussion knowledge index (CKI) and concussion attitudes/behaviours index (CAI) regarding RTP. Results: On average, $62 \%$ (JAHS) and $60 \%$ (SAC) of the CKI questions were answered correctly. JAHS participants correctly identified $66 \%$ of concussion symptoms, similarly to the SAC participants $(63 \%)$, rendering similar $(\mathrm{p}=0.37)$ overall CKI scores when comparing the two groups. The CAI questions yielded similar ( $\mathrm{p}=0.98$ ) results between the groups, reporting safe responses in $66 \%$ (JAHS) and $67 \%$ (SAC) of the items.

Discussion and conclusion: Junior and senior South African amateur rugby players lacked approximately one-third of essential concussion knowledge, which may lead to a display of unsafe attitudes/behaviours to concussion and RTP. Further research is warranted to inform educational programmes on concussion among rugby players.

Keywords: post-concussion syndrome, rugby union, return to play, safety, South Africa

S Afr J Sports Med 2017;29:1-6. DOI: 10.17159/2078-516X/2017/v29i0a1942

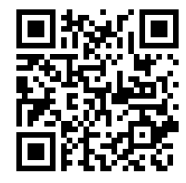

Rugby players are exposed to a higher risk of injury than participants in other sports, as players may legitimately be involved in collision incidents. ${ }^{[1]}$ A recent review reported that head injuries, including concussions, are the most frequently reported injuries in professional rugby. ${ }^{[2]}$ Concussion is defined as a 'traumatically induced transient disturbance of brain function which involves a complex pathophysiological process' ${ }^{\left[{ }^{3]}\right.}$ Potentially dangerous long-term side-effects, such as neurological deficits and chronic traumatic encephalopathy, explain why the topic of concussion is covered extensively in medical and lay media. ${ }^{[4]}$ Concussion in rugby is most commonly noted at community/amateur level, with a rate of 2.08 concussions per 1000 player match-hours..$^{[5]}$ This is followed by schoolboy level and elite level, with respective rates of 0.62 and 0.40 concussions per 1000 player match-hours. ${ }^{[5]}$

Most of South Africa's rugby playing population consists of amateur players. Only a limited proportion of amateur clubs and schools offer medical assistance - which is limited to matches - mostly due to a lack of funding. ${ }^{[6]}$ Therefore the focus of the BokSmart programme in South Africa currently leans towards educating coaches and referees to recognise concussion signs and symptoms and to remove concussed players from the field. ${ }^{[6]}$ In a country where medical assistance on the field during a rugby match is scarce, the players themselves can play a pivotal role in reporting possible concussions to their coach or the referee.

Only a few studies have researched rugby players' knowledge of concussion. ${ }^{[7-9,11,12]}$ Less than half of a group of New Zealand high school rugby players knew about concussion, while only $22 \%$ of players waited to be medically cleared for return to play (RTP) after being diagnosed with concussion. ${ }^{[7]}$ Research among Italian amateur rugby players showed that $39 \%$ of these players had never been informed about concussion. ${ }^{[8]}$ Similar results were noted among 127 subelite South African rugby players, where less than half of the players waited until fully recovered before returning to full participation in rugby. ${ }^{[9]}$

Currently, no rugby safety management programme focuses on concussion education among South African rugby players. Only a single study, to date, has investigated knowledge of concussion and RTP attitudes among South African rugby players and therefore further research in this field of study is warranted. [9] The aim of this study was to evaluate the concussion knowledge and attitudes to RTP of South African amateur rugby players in order to provide information for future concussion education programmes. Based on anecdotal evidence and the study by Walker, ${ }^{[9]}$ it was found that amateur South African rugby players displayed insufficient knowledge concerning concussions.

\section{Methods}

Permission to conduct this study was granted by the South 
African Rugby Union and the Gauteng Department of Education, while ethical approval was obtained from the Institutional Ethics Committee of the University of the Free State. A total of 294 South African amateur rugby players participated in this cross-sectional study and were recruited from high schools and clubs in the Gauteng province. From the more than 200 schools in Gauteng who were actively playing rugby at the time of the research, 30 schools were randomly selected and approached to participate in the study. From these schools, seven agreed to participate. All Gauteng province-based rugby clubs with contact details available on the internet or via the specific rugby unions were contacted for inclusion in the study. Six rugby clubs finally agreed to partake in the study.

The study sample was divided into two groups of players, namely, those from junior amateur high school (JAHS) $(n=216$, aged 13-19 years) and those from senior amateur club (SAC) ( $n=78$, aged 17-28 years). All participants had to be male, South African citizens, and proficient English-speaking rugby players. Participants in the SAC group had to be registered at a club in the Gauteng Province during the 2015 club rugby season, and must have played at least one game for the club that season in either the under-21 or the over-21 age groups. Participants in the JAHS group had to have been playing at under-14, -16 or -18 age levels during the 2015 season, be enrolled at a Gauteng school, and have played at least one game for the school during the 2015 season.

Participants completed a modified Rosenbaum Concussion Knowledge and Attitudes Survey - Student Version (RoCKAS-ST) questionnaire ${ }^{[10]}$ to assess their knowledge and attitudes/behaviour regarding concussion injuries and RTP. The original questionnaire consisted of the Concussion Knowledge Index (CKI), Concussion Attitudes Index (CAI) and the RoCKAS concussion symptom recognition checklist. ${ }^{[10]}$ The modified version replaced the RoCKAS concussion symptom recognition checklist with a 16symptom checklist which increased the reliability and validity of the questionnaire. ${ }^{[10]}$ Descriptive statistics were used to summarise continuous data with means and standard deviations or medians and quartiles $\left(\mathrm{Q}_{1}\right.$ and $\left.\mathrm{Q}_{3}\right)$ as appropriate. Frequencies and percentages were calculated for categorical data while significance was set at $\mathrm{p}<0.05$ for comparative analyses.

\section{Results}

At the time of the study, 33\% from the JAHS and $42 \%$ from the SAC groups' participants reported a previous history of having sustained a concussion. The largest proportion JAHS participants were front row players $(26 \%)$ while the largest proportion of SAC participants were in the back three $(27 \%)$ (Fig. 1). The JAHS group had a median rugby playing experience of eight years $\left(Q_{1}=7 ; Q_{3}=10\right)$ compared to the $S A C$ group with a median of 10 years $\left(Q_{1}=6 ; Q_{3}=15\right)$. Sixty percent of the SAC participants were under-21 players.

Only $46 \%(n=133)$ from the total sample had previously received information on concussion. A higher number of participants in the SAC group were more informed on concussion $(59 \%, n=44)$ compared to participants in the JAHS group $(41 \%, n=89)$. Healthcare professionals $(\mathrm{HCP})$ played the biggest role as the source of information to $42 \%(n=43)$ of all participants. Those who had previously received concussion information reported HCPs as their information source. The JAHS group, specifically, received the majority of their information on concussion from HCPs $(47 \%, n=34)$, while the SAC group received their information on concussion from the schools $(33 \%, \mathrm{n}=10)$ they had previously attended (Fig. 2).

\section{$\square$ JAHS $\square$ SAC}

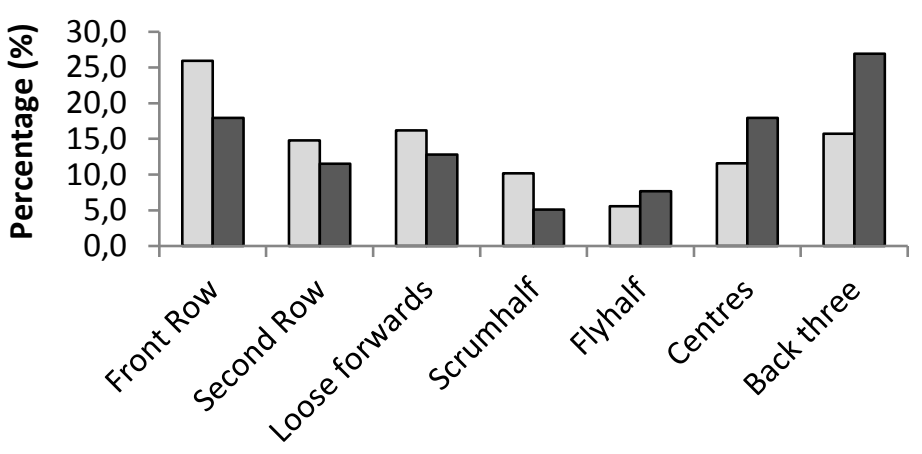

Player positions

Fig. 1. Player position distributions of the junior amateur high school (JAHS, $n=216)$ and the senior amateur club $(S A C, n=78)$ participants.

\section{口JAHS $\square$ SAC}

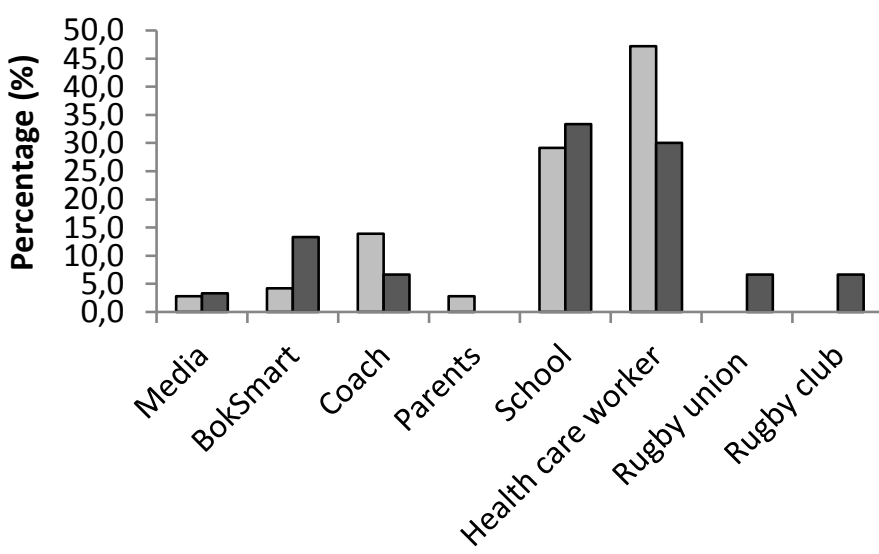

Fig. 2. Sources of information on concussion received by the junior amateur high school (JAHS, $n=72)$ and the senior amateur club (SAC, $n=30$ ) participants.

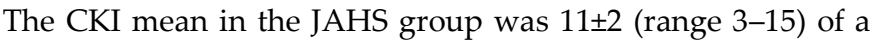
maximum score of 17 points, while the SAC group showed a mean of $10 \pm 2$ (range 4-14) also of a maximum score of 17 points. Participants in the JAHS group, on average, identified $62 \%$ of the CKI questions correctly, which was similar compared to the $60 \%$ identified correctly by the SAC participants ( $\mathrm{p}=0.37$ ).

Most of the knowledge questions were correctly answered by less than $80 \%$ of the JAHS participants (Table 1 ). The most common misconception was that a concussion could be identified via brain imaging; only $23 \%(n=50)$ of the JAHS group and $19 \%(n=14)$ of the SAC participants answered this question correctly. Both groups had a misconception about the mechanism of the concussion injury, as $63 \%(n=133)$ of the JAHS 
and $57 \%(n=44)$ of the SAC participants believed that a concussion could only be sustained by a direct hit to the head. Interestingly, only $32 \%$ of the JAHS group knew that multiple concussions can have a negative long-term effect on a player's health and well-being (Table 1, item 14). When the same concept was tested in a scenario they could relate to, then $81.9 \%$ of the JAHS group answered correctly. Similarly, the SAC group's correct answer percentage increased by $15.5 \%$ when the concept was tested in a playing scenario they were familiar with.

On average, participants in the JAHS group identified the symptoms of concussion correctly $66 \%$ of the time compared to $63 \%$ by SAC participants. True symptoms of concussion that were unfamiliar to the majority of JAHS and SAC participants included sleep disturbances, loss of

Table 1. Concrete statements and scenarios evaluating the Concussion Knowledge Index (CKI) of the JAHS and SAC groups.

True Statements
1. There is a possible risk of death if a second
concussion occurs before the first one has healed.
2. People who had one concussion are more likely
to have another concussion.
3. Symptoms of a concussion can last several
weeks.
4. After 10 days, symptoms of a concussion are
usually completely gone.
5. Concussions can sometimes lead to emotional
disruptions.
6. An athlete who gets knocked out after getting
a concussion is experiencing a coma.

\section{False Statements}

7. In order to be diagnosed with a concussion you

\begin{tabular}{cc}
\multicolumn{2}{c}{$\%$ Correct } \\
JAHS & SAC \\
82 & 77 \\
66 & 48 \\
80 & 80 \\
60 & 53 \\
72 & 66 \\
38 & 37
\end{tabular}
have to be knocked out.

8. A concussion can only occur if there is a direct hit to the head.

9. Being knocked unconscious always causes permanent damage to the brain.

10. Sometimes a second concussion can help a person remember things that were forgotten after the first.

11. After a concussion occurs, brain imaging (CAT scan, MRI, X-ray etc.) typically show visible physical damage (bruise, blood clot) to the brain. 12. If you receive one concussion and you have never had a concussion before, you will become less intelligent.

13. After a concussion, people can forget who they are and not recognise others but be perfect in every other way.

14. There is rarely a risk to long-term health and well-being from multiple concussions.

\section{Scenarios}

1.1. A single concussion will affect a player's health and well-being negatively

1.2. A history of multiple concussions will affect a player's health and well-being negatively

2. Playing with concussion symptoms will not affect a player's performance
Table 2. Concussion symptom identification - capabilities of the JAHS and SAC groups.

\begin{tabular}{lcc}
\hline \multicolumn{1}{c}{ Symptom } & \multicolumn{2}{c}{ \% Correct } \\
\hline The following are symptoms & JAHS (n=216) & SAC (n=78) \\
Amnesia (memory loss) & 69 & 56 \\
Blurred vision & 73 & 72 \\
Confusion & 77 & 69 \\
Dizziness & 84 & 71 \\
Headache & 84 & 77 \\
Loss of consciousness & 47 & 56 \\
Nausea & 57 & 51 \\
Sleep disturbances & 38 & 47 \\
& & \\
The following are not symptoms & & \\
Abnormal sense of smell & 92 & 88 \\
Abnormal sense of taste & 88 & 87 \\
Black eye & 91 & 86 \\
Chest pain & 83 & 91 \\
Nosebleed & 73 & 85 \\
Numbness/tingling in upper & 79 & 76 \\
extremity & & \\
Sharp burning pain in neck & 85 & 65 \\
Weakness of neck range of motion & 59 & \\
& & \\
\hline JAHS; junior amateur high school, SAC; senior amateur club &
\end{tabular}

consciousness and nausea (Table 2). Dizziness and headaches were the symptoms that both the JAHS and SAC groups were most familiar with.

Similar $(\mathrm{p}=0.98)$ mean CAI scores were noted for both groups (Table 3). The CAI mean in the JAHS group was $57 \pm 9$ (range 36-75) compared to $56 \pm 11$ (range 20-75) reported among the SAC participants. The CAI questions were scored on a Likert scale and categorised into "safe", "neutral" and "unsafe" responses. Participants in the JAHS group had a mean safe response of $66 \%$ compared to $67 \%$ for SAC participants when analysing the total number of safe, neutral and unsafe responses across the various statements. The majority of JAHS $(79 \%, n=169)$ and SAC participants $(85 \%, n=63)$ agreed that a concussed player must be prevented from returning to play by the coach, even if it means losing the game (Table 3, scenario 1.1).

Both the JAHS and SAC participants showed unsafe responses to the question of sports participation while concussed. Only $55 \%$ of JAHS $(n=115)$ and SAC $(n=43)$ participants reported that they themselves would take the decision to refrain from participation in sport while they still had symptoms that resulted from a concussion (Table 3, statement 1$)$. Only $66 \%(n=141)$ of JAHS participants believed that a physiotherapist rather than the player should decide when it is safe to RTP. The SAC participants showed similar results, as only $53 \%(n=40)$ agreed that the player should not be the one making the RTP decision. A slight decline in safe RTP responses ( $1 \%$ for JAHS and $4 \%$ for SAC participants) towards a hypothetical scenario were noted when playoff games were in question compared to the first match of the season (Table 3, scenario 2). However, when distinguishing between those who "strongly disagreed" and those who just "disagreed", only $28 \%$ of the SAC participants strongly disagreed that the player 
Table 3. Concrete statements and scenarios evaluating the Concussion Attitude Index (CAI) of the JAHS and SAC groups.

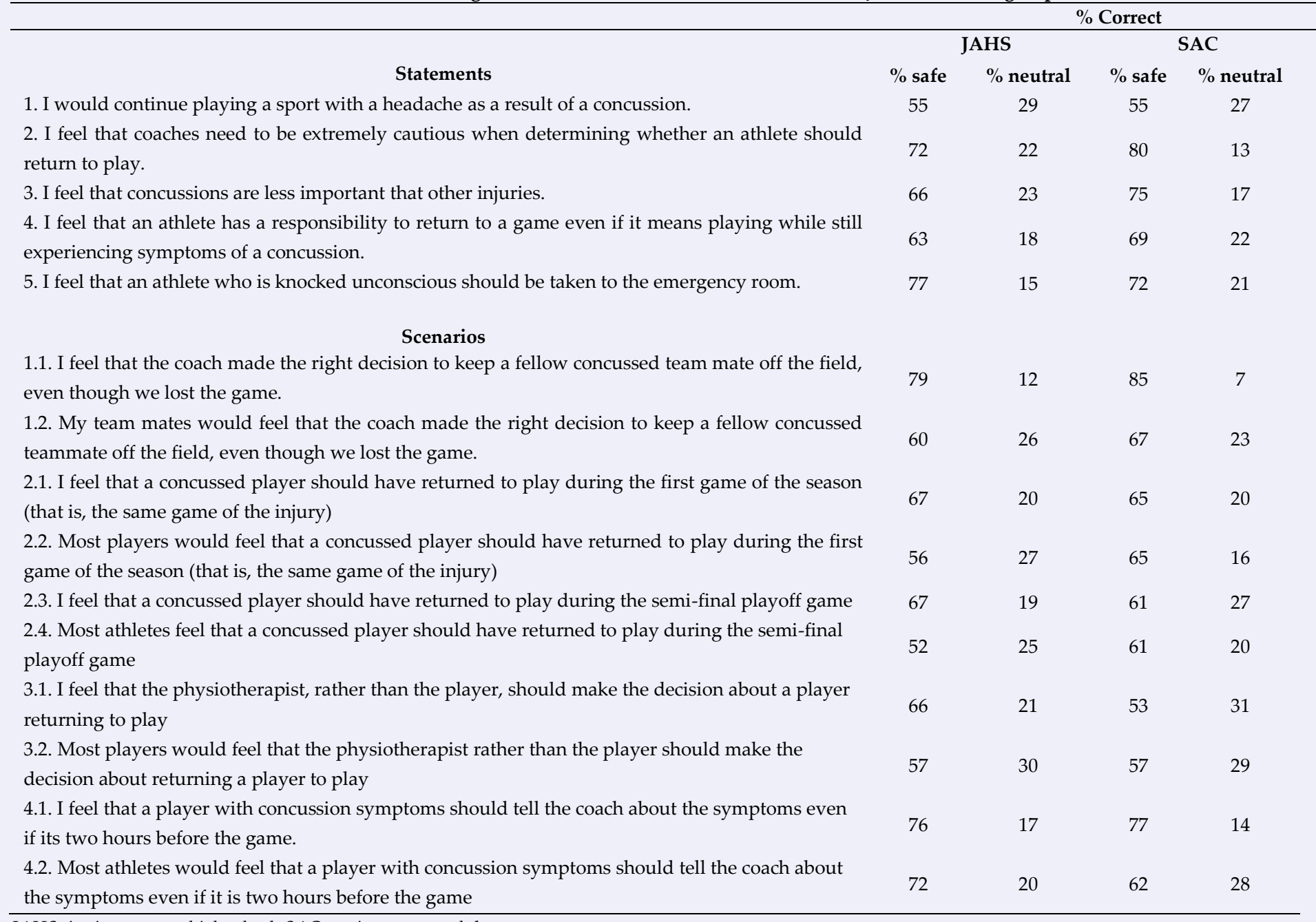

JAHS; junior amateur high school, SAC; senior amateur club

should RTP during a playoff game compared to $40 \%$ who strongly disagreed when it was the first match of the season.

\section{Discussion}

As far as could be established, this was the first study including both junior and senior amateur rugby players, comparing data relating to concussion knowledge and attitudes/behaviours. This study sample (294 participants) is substantially larger when compared to other studies that investigated concussion knowledge among rugby players. ${ }^{[8,9,11,12]}$ Only $46 \%$ of the total study sample had previously received information on concussion, which is marginally more than the 39\% informed participants reported for Italian rugby players. ${ }^{[8]}$ A better-informed sample was reported by Sye et al., ${ }^{[7]}$ where $61 \%$ of their participants had received concussion information. The fact that more SAC (59\%) compared to JAHS (41\%) participants from this study reportedly received concussion information may possibly be attributed to the fact that they also had a higher reported incidence of concussion and player years and, therefore, possibly greater exposure to concussion management and associated concussion information. Due to the fact that the participants from this study were amateur school and club players not necessarily playing in a specific position, with a median rugby playing experience of eight and 10 years respectively, the participants were more likely to have changed player positions during their rugby careers. This would make inferences on the link between player positions and concussion histories speculative at most, since the participants reported their concussion histories irrespective of when they occurred.

Similarly to previous studies, ${ }^{[8]}$ HCPs played the biggest role as a source of information to $42 \%$ of the informed participants. Schools were also identified as a noteworthy source of information (Fig. 2). These should be investigated in future research as a possible avenue for concussion education among rugby players who are at different developmental stages compared to the coaches and referees targeted by BokSmart. It must be acknowledged that teammates could inherently be part of the school source for concussion information and should also be considered in future research.

As BokSmart focuses on educating coaches and referees on concussion, ${ }^{[6]}$ it was expected that coaches would have played a pronounced role as a source of concussion information. However, only $14 \%$ of the JAHS and $7 \%$ of the 
SAC participants reported coaches as their source of information on concussion. Considering the fact that teachers often fill the role of rugby coaches, it must be acknowledged that some JAHS participants included teachers as opposed to coaches as a school source of information. In contrast, the low occurrence of coaches conveying concussion information reported by the SAC players could not be explained and seems to imply that coaches are not a significant source of concussion information among senior amateur rugby players. A third (33\%) of SAC participants also reported schools as their main source of concussion information, due to the fact that $60 \%$ of the SAC participants were under-21 players and had therefore recently attended school.

Similar to the findings of Boffano et al. ${ }^{[8]}$ the media were also identified as playing a limited role as a source of concussion information. However, social media are widely used among all ages and their role should be investigated in future research on concussion education.

Participants in the JAHS group on average identified 62\% of the CKI questions correctly, while the SAC group had an average of $60 \%(p=0.37)$. Considering that $59 \%$ of the SAC participants had previously received information about concussion, the percentage of informed participants seemed to be closely related to the mean percentage of correctly answered CKI questions. However, this speculative relationship between information received and CKI scores was not evident among the JAHS group, who managed to obtain a mean of $62 \%$ of the CKI answers correct, even though only $41 \%$ of them had previously received information on concussion.

The most common misconception among participants in both groups was that a concussion could be identified via brain imaging, showing physical brain damage, which is in keeping with other studies.[1] Considering that patients cannot refer themselves for brain imaging, the practical implication of this finding among rugby players may not be of major concern. However, the effect of this misconception on a player's self-driven RTP decisionmaking is unknown and should be considered in future research.

Another apparent misconception is displayed in the mere $37 \%$ of JAHS participants and $43 \%$ of SAC participants who indicated that a concussion can be sustained via a hit anywhere on the body, and not only on the head. Similar results have previously been reported, where only $47 \%$ of a study sample could correctly identify that head impact was not the only way to sustain a concussion. ${ }^{[1]}$ This is a further motivation for education among rugby players to enable them to link preceding mechanisms of sustaining a concussion to the potential concussion symptoms displayed by teammates.

Among the JAHS and SAC participants, a headache was the most common correctly identified concussion symptom. In comparison, Boffano et al. ${ }^{[8]}$ reported headaches as the sixth most commonly identified concussion symptom. These authors reported symptoms of nausea, vomiting, memory loss, dizziness and loss of consciousness as symptoms more frequently identified by Italian rugby players. ${ }^{[8]}$ Dizziness was also frequently identified correctly in the current study, with $84 \%$ of JAHS participants and $71 \%$ of SAC participants providing correct identification. In contrast to Boffano et al. ${ }^{[8]}$ the current study showed a low rate of correct identification of memory loss as a symptom of concussion; only $58 \%$ of SAC participants could correctly identify this symptom.

Even though the JAHS participants had better concussion knowledge, they displayed more unsafe attitudes or behaviours regarding concussion and RTP compared to the SAC participants. In keeping with other health-related studies, such as knowledge of the risks of smoking which does not necessarily govern cessation habits, ${ }^{[13]}$ the results from this study support other concussion studies, where the use of increased concussion knowledge as a predictor of the effectiveness of concussion educational tools has been criticised. ${ }^{[14]}$ Kroshus et al. argued that concussion knowledge only indicates if the individual paid attention to the information received, and not necessarily whether it is indicative of improved in-season reporting behaviours. ${ }^{[14]}$ This view is echoed by Kurowski et al. [15] who state that improved self-report behaviours were not associated with previous concussion education or better knowledge about concussion. In order to obtain effective behavioural change, Kroshus et al. ${ }^{[14]}$ propose adding real life simulation to educational programmes in order to help individuals make safe decisions.

Both the JAHS and SAC participants displayed unsafe attitudes to self-driven RTP approaches while concussed; only $55 \%$ of all participants reported that they would stop their participation in sport while experiencing concussion symptoms. This is alarming, considering that the majority of participants (JAHS $=82 \%, \quad \mathrm{SAC}=77 \%$ ) understood the potential risk of death when a second concussion is sustained while already concussed. This discrepancy could possibly indicate a misconception among players that the presence of some concussion symptoms does not necessarily constitute a diagnosis of being concussed. This conception should be investigated in future research. Furthermore, this finding emphasises a need for concussion education among players in order to address possible misconceptions and promote safer attitudes/behaviours in concussion and RTP. Increasingly unsafe attitudes/behaviours during playoff versus the first game of the season were seen among the SAC participants. $\mathrm{O}^{\prime}$ Connell and Molloy ${ }^{[12]}$ found similar unsafe attitudes in their research, as $75 \%$ of their participants reported that they would continue to play with a concussion in important games. They further reported that players showed these unsafe behaviours as they did not want to let the team down. ${ }^{[1]}$

Certain limitations were identified during the course of the present study. Sample size and selection were affected by the time of year data collection occurred, which resulted in fewer under-18 level players included in the JAHS group due to the lack of scholars available for testing. A two-week period was given for data collection at school level, giving rise to the possibility of questionnaire content leaking, with 
associated peer influence among scholar participants at different age levels.

\section{Conclusion}

Both junior and senior South African amateur rugby players displayed insufficient knowledge of concussion and unsafe attitudes/behaviours regarding concussion and RTP, with no statistically significant differences between their mean CKI and the mean CAI scores. Unsafe attitudes were more evident during pressure situations, as participants showed higher unsafe responses towards RTP when playoff games were involved. This provides motivation for further implementation of concussion education programmes and their scope among amateur South African rugby players through the BokSmart rugby safety programme. Future research should focus on incorporating other geographical regions of South Africa in order to improve generalisability of the findings, and implement population-specific concussion education programmes among all role players. The role of social factors in unsafe concussion attitudes/behaviours, despite the availability of information, should also be investigated for inclusion in concussion education programmes.

Conflict of interest: The authors have no conflict of interest to declare. No funding was received from any funding agencies in the public, commercial or non-profit sectors.

\section{References}

1 Carter M. The unknown risks of youth rugby. BMJ 2015;350:h26. doi: 10.1136/bmj.h26 [PMID: 25566788]

2 Kaux JF, Julia M, Delvaux F, et al. Epidemiological review of injuries in rugby union. Sports 2015;3:21-29.doi: 10.3390/sports3010021

3 Harmon KG, Drezner JA, Gammons M, et al. American Medical Society for Sports Medicine position statement: concussion in sport. Br J Sports Med 2013;47(1):15-26. doi: 10.1136/bjsports-2012-091941 [PMID: 23243113]

4 Raftery M. Concussion and chronic traumatic encephalopathy: International Rugby Board's response. Br J Sports Med 2014;48(2):79-80. doi: 10.1136/bjsports-2013093051 [PMID: 24096899]
5 Gardner AJ, Iverson GL, Williams WH, et al. A systematic review and meta-analysis of concussion in rugby union. Sports Med 2014;44(12):1717-1731. doi: 10.1007/s40279-014-0233-3 [PMID: 25138311]

6 BokSmart. What is BokSmart.. Available from: http://boksmart.co.za/content/what-is-boksmart (accessed 15 August 2015).

7 Sye G, Sullivan SJ, McCrory P. High school rugby players' understanding of concussion and return to play guidelines. $\mathrm{Br}$ J Sports Med 2006;40(12):1003-1005. doi 10.1136/bjsm.2005.020511 [PMID:17124109]

8 Boffano P, Boffano M, Gallesio C, et al. Rugby players' awareness of concussion. J Craniofac Surg 2011;22(6):20532056.

9 Walker S. Concussion knowledge and return-to-play attitudes among subelite rugby union players. S Afr J Sports Med 2015;27(2):50-54.

10 Williams J. Concussion knowledge and attitudes in English football (soccer). Master's Thesis. Georgia, USA. Georgia Southern University; 2013. Available from: Electronic Theses and Dissertations (accessed 12 January 2015).

11 Baker JF, Devitt BM, Green J, et al. Concussion among under20 rugby union players in Ireland: incidence, attitudes and knowledge. Ir J Med Sci 2013;182(1):121-125. doi: 10.1007/s11845-012-0846-1 [PMID: 22898834]

12 O'Connell E, Molloy MG. Concussion in rugby: knowledge and attitudes of players. Ir J Med Sci 2016;185(2):521-528. doi: 10.1007/s11845-015-1313-6 [PMID:26026952]

$13 \mathrm{Xu} \mathrm{X}$, Liu L, Sharma M, et al. Smoking-related knowledge, attitudes, behaviours, smoking cessation idea and education level among young adult male smokers in Chongqing, China. Int J Environ Res Public Health 2015;12(2):2135-2149. doi:10.3390/ijerph120202135.

14 Kroshus E, Baugh CM, Daneshvar DH, et al. Concussion reporting intention: a valuable metric for predicting reporting behaviour and evaluating concussion education. Clin J Sport Med 2015;25(3):243-247. doi: 10.1097/JSM.0000000000000137.

15 Kurowski B, Pomerantz WJ, Schaiper C, et al. Factors that influence concussion knowledge and self-reported attitudes in high school athletes. J Trauma Acute Care Surg 2014;77(3 Suppl 1):S12-S17. doi: 10.1097/TA.0000000000000316 [PMID 25153048] 